\section{Quantitative investigations of supported metal catalysts by ASAXS}

\section{Stefano Polizzi, ${ }^{a *}$ Pietro Riello, ${ }^{a}$ Günter Goerigk ${ }^{b, c}$ and Alvise Benedetti ${ }^{\mathrm{a}}$}

\author{
a Dipartimento di Chimica Fisica, Università Ca' Foscari di \\ Venezia, Via Torino 155/b, 30170 Venezia-Mestre, Italy, \\ ${ }^{b}$ Institut für Festkörperforschung Forschungszentrum Jülich \\ $\mathrm{GmbH}$, Germany, and ${ }^{C} H A S Y L A B$ (DESY), Hamburg, Germany. \\ E-mail:polizzi@unive.it
}

Limits and potentiality of anomalous small-angle X-ray scattering for the investigation of supported metal catalysts are discussed. The different sources of statistical errors are illustrated using two catalysts containing metals with absorption edges at very different energies ( $\mathrm{Au}$ and $\mathrm{Pd}$ ). Optimized experimental conditions and measuring strategy are proposed, and data-evaluation methods for obtaining quantitative reliable results are suggested. By this method, Au content as low as $0.2 \mathrm{wt} \%$ and $\mathrm{Pd}$ content of $3 \mathrm{wt} \%$ could be investigated with success. The detection limits for palladium are higher, mainly due to its smaller electronic contrast.

Keywords: ASAXS; methods; catalysis.

\section{Introduction}

Anomalous small-angle X-ray scattering (ASAXS) has been increasingly used over the last few years to study supported metal catalysts (Haubold \& Wang, 1995; Haubold et al., 1997; Benedetti et al., 1997, 1999; Rasmussen et al., 2000; Polizzi et al., 2001) because of its ability to determine the size distribution of the active particles on a very convenient scale range, from nanoparticles of several tens of nanometres down to clusters of $1 \mathrm{~nm}$. These kinds of catalysts are composed of an active metal phase dispersed in a porous support. The efficiency of the catalyst is increased by a good dispersion of the metal (no agglomeration or sintering) and by very small particles, since in this way the metal surface exposed to reagents is maximized.

ASAXS has proven to be able to provide essential quantitative information on these materials and is now considered an important technique in this field. However, the ASAXS data result from a small difference in the scattering at two energies, which is dependent on the particular metal and is obviously proportional to the amount of metal contained in the sample. Since industrial catalysts usually contain low metal fractions, the study of the detection limits of the ASAXS technique is of great interest.

In the present investigation, two cases are illustrated in detail: one catalyst containing $\mathrm{Au}$ and one containing Pd. Taking metals with absorption edges at very different energies was considered to represent a good choice to test the possibilities of ASAXS. The different sources of statistical errors are investigated and possible experimental improvements and appropriate evaluation methods are shown, with the aim of understanding, and possibly extending, the limits of the technique.

\section{Theoretical background}

For the simple case of homogeneous spherical metal particles with radius $r$ in a homogeneous matrix, the small-angle scattering cross section per sample volume, $V_{\mathrm{s}}$, can be written as (neglecting particle interference scattering) (Haubold \& Wang, 1995; Haubold et al., 1996)

$$
\frac{\mathrm{d} \sigma}{\mathrm{d} \Omega_{\mathrm{Me}}}(h, E)=\Delta \rho^{2}(E) \frac{N_{\mathrm{Me}}}{V_{\mathrm{s}}} \int_{0}^{\infty} P(r) V^{2}(r) S^{2}(h, r) \mathrm{d} r,
$$

where $N_{\mathrm{Me}}$ is the number of metal particles and $h=4 \pi \sin \theta / \lambda$ is the modulus of the scattering vector with wavelength $\lambda$ and scattering angle $2 \theta . V(r)$ is the volume of a particle and $P(r)$ is the size distribution. $S(h, r)$ denotes the scattering function of a spherical particle (Guinier \& Fournet, 1955). $\Delta \rho(E)$ is the energy-dependent electron density contrast between the metal particles and the surrounding matrix,

$$
\Delta \rho(E)=\left|d_{\mathrm{Me}} f_{\mathrm{Me}}(E)-d_{\mathrm{M}} f_{\mathrm{M}}(E)\right|,
$$

where $d_{\mathrm{Me}}$ and $d_{\mathrm{M}}$ are the average atomic densities of the metal particles and of the matrix, respectively. $f_{\mathrm{Me}}$ are the atomic scattering factors of the metal, which in general is energy-dependent,

$$
f_{\mathrm{Me}}(E)=Z_{\mathrm{Me}}+f_{\mathrm{Me}}^{\prime}(E)+i f_{\mathrm{Me}}^{\prime \prime}(E),
$$

where $Z_{\mathrm{Me}}$ denotes the number of electrons of the metal atoms and $f^{\prime}(E)$ and $f^{\prime \prime}(E)$ are the so-called anomalous dispersion corrections. In the vicinity of the absorption edges of the metal, the atomic scattering factor of the metal particle shows strong variation (see, for example, Figs. 1 and 2), while the atomic scattering factor of the matrix atoms remains almost constant $\left[f_{\mathrm{M}}(E) \simeq\right.$ constant $]$.

Applied to the situation of a sample containing metal particles in a matrix with a porous structure, for instance in a carbon or silica support, SAXS studies with a fixed X-ray energy, e.g. the characteristic radiation from an X-ray tube, do not allow one to separate the scattering of the metal particles from the scattering of the support material, because the pores represent a third phase (with zero electron density). In this case, the total scattering is a superposition of only two contributions $\dagger$ and is written as follows (Haubold \& Wang, 1995; Haubold et al., 1996),

$$
\frac{\mathrm{d} \sigma}{\mathrm{d} \Omega_{\text {total }}}(h, E)=\frac{\mathrm{d} \sigma}{\mathrm{d} \Omega_{\mathrm{Me}}}(h, E)+\frac{\mathrm{d} \sigma}{\mathrm{d} \Omega_{\mathrm{M}}}(h)
$$

Owing to the weak energy dependence of $f_{\mathrm{M}}$, which enters into $\mathrm{d} \sigma / \mathrm{d} \Omega_{\mathrm{M}}$, the small-angle scattering of the matrix remains almost unchanged with energy, while the small-angle scattering of the metal particles changes strongly at energies near the absorption edge of the metal. Thus, the scattering of the metal particles can be obtained by subtraction of the small-angle scattering measured at the two energies $E_{1}$ and $E_{2}$,

$$
\begin{aligned}
\frac{\mathrm{d} \sigma}{\mathrm{d} \Omega_{\text {total }}}\left(h, E_{1}\right)-\frac{\mathrm{d} \sigma}{\mathrm{d} \Omega_{\text {total }}}\left(h, E_{2}\right)= & {\left[\Delta \rho^{2}\left(E_{1}\right)-\Delta \rho^{2}\left(E_{2}\right)\right] \frac{N_{\mathrm{Me}}}{V_{\mathrm{s}}} } \\
& \times \int_{0}^{\infty} P(r) V^{2}(r) S^{2}(h, r) \mathrm{d} r .
\end{aligned}
$$

Thus, the size distribution of metal particles can be easily obtained by using an analytical function for $P(r)$ and fitting the calculated scat-

\footnotetext{
$\dagger$ The cross term describing mutual correlations is neglected for the following reason. In metal-supported catalysts obtained by impregnation, metal particles are mostly deposited in pores with sizes significantly larger than the metal particles, since such pores are more easily filled by the liquid solution. In this case, if one imagines the metal particles distributed over the inner surface of the pores, the strong cross-correlation between pores and metal particles would give contributions to the scattering only at very small angles, outside the visibility range of the experiment. The cross-correlation of neighbouring pores would appear at even lower scattering angles.
} 
tering intensities [the integral in (5)] to the experimental data by means of an optimization procedure.

If the experimental scattering intensities are scaled in absolute electron units, further quantitative results can be obtained from the scale factor $K=\left[\Delta \rho^{2}\left(E_{1}\right)-\Delta \rho^{2}\left(E_{2}\right)\right] N_{\mathrm{Me}} / V_{\mathrm{s}}$, which is an output of the optimization procedure. If the difference in electronic contrast is known, the volume fraction of the metal phase can then be determined, since $V_{f}=N_{\mathrm{Me}}\langle V\rangle / V_{\mathrm{s}}$, and the average particle volume, $\langle V\rangle$, can be calculated from the best-fit particle distribution $P(r)$ by $\langle V\rangle=\int P(r)(4 / 3) \pi r^{3} \mathrm{~d} r$. Furthermore, if the densities of the sample $\left(d_{\mathrm{s}}\right)$ and of the metal particles $\left(d_{\mathrm{m}}\right)$ are known, the obtained $V_{f}$ can be compared with the weight fraction, $V_{\mathrm{w}}$, obtained by atomic spectroscopy $\left(V_{\mathrm{w}}=\mathrm{V}_{f} d_{\mathrm{m}} / d_{\mathrm{s}}\right)$, and can serve as a consistency test for the ASAXS evaluation procedure.

Yet, in the case of supported catalysts, the electron density contrast terms $\Delta \rho^{2}\left(E_{i}\right)$ are badly defined, because of the three-phase nature of these systems (metal, support, pores). In fact, while for the metal the electron density of the bulk metal can be used, the electron density of the matrix will be something between that of the bulk matrix and zero (pores). In some cases, such as noble metals on carbon, where the electronic density difference between metal and matrix is very high,

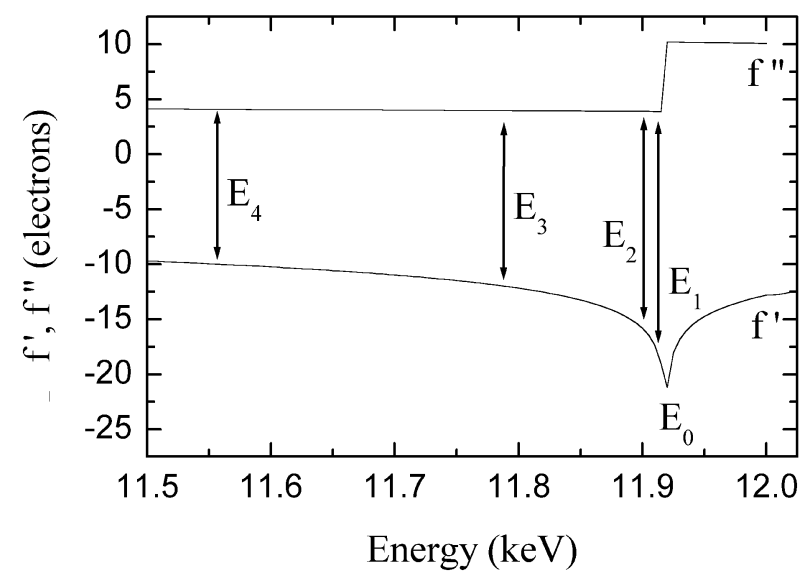

Figure 1

Energy-dependent anomalous dispersion corrections of the atomic scattering factor of $\mathrm{Au}, f^{\prime}$ and $f^{\prime \prime}$, as a function of energy. The arrows show the energies at which measurements have been carried out (see Table 1 for energy values).

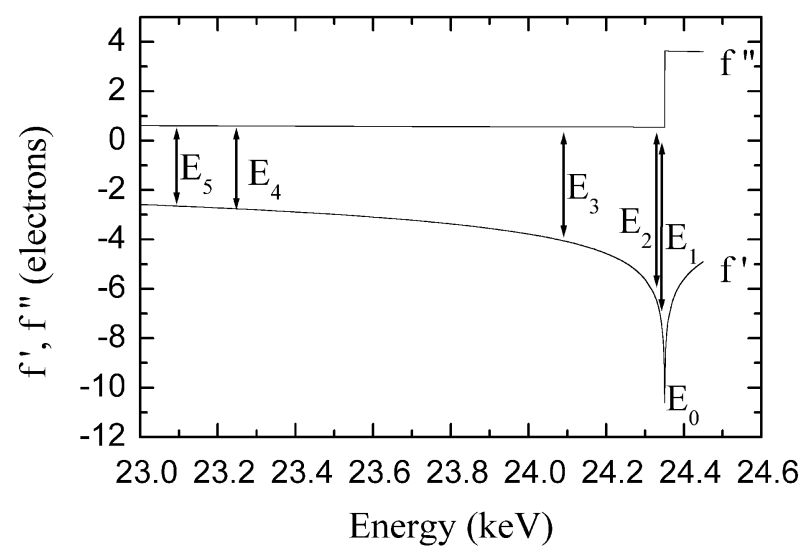

Figure 2

Energy-dependent anomalous dispersion corrections of the atomic scattering factor of $\mathrm{Pd}, f^{\prime}$ and $f^{\prime \prime}$, as a function of energy. The arrows show the energies at which measurements have been carried out (see Table 2 for energy values). this ambiguity does not lead to significant differences, so that Haubold \& Wang (1995) could neglect the influence of the surrounding carbon. On the contrary, in the case of $\mathrm{Pd} / \mathrm{SiO}_{2}$ catalysts, different choices for the electron density of the matrix yield significant differences in the electron contrast and hence in the estimate of $V_{f}$.

Therefore, while anomalous scattering allows one to isolate the scattering of the metal phase and to overcome the difficulties related to the high porosity of the support, it does not always solve the problem of the quantitative determination of the metal content. However, the two limit cases can be worked out: $(a)$ metal particles embedded in a bulk matrix, or $(b)$ metal particles inside pores. In the first case the electron density of the bulk support (carbon or silica) is taken as a matrix, whereas in the second case a zero electron density is used. In this way, two values of $V_{f}$ are obtained, which define a range where the actual value is predicted to be.

\section{Experimental}

\subsection{Sample preparation}

For the Au sample, a commercial active carbon with a surface area of $1200 \mathrm{~m}^{2} \mathrm{~g}^{-1}$ and a pore volume of $0.60 \mathrm{ml} \mathrm{g}^{-1}$ has been used as support. The catalyst was obtained by impregnation with an aqueous solution of $\mathrm{HAuCl}_{4}$ and was reduced by $\mathrm{HCOOH}$ at $353 \mathrm{~K}$. The $\mathrm{Au}$ content determined by atomic spectroscopy was $0.2 \mathrm{wt} \%$.

The $\mathrm{Pd}$ sample was prepared by impregnation of $\mathrm{SiO}_{2}$ (Akzo Chemie F22; surface area $400 \mathrm{~m}^{2} \mathrm{~g}^{-1}$; particle size range $\left.100-150 \mu \mathrm{m}\right)$ with aqueous solutions of $\mathrm{Na}_{2} \mathrm{PdCl}_{4}$. After drying at $383 \mathrm{~K}$ for $18 \mathrm{~h}$, samples were calcinated at $773 \mathrm{~K}$ for $1 \mathrm{~h}$, then reduced at $623 \mathrm{~K}$ for $2 \mathrm{~h}$ and passivated $\left(5 \% \mathrm{O}_{2}\right.$ in $\left.\mathrm{Ar}\right)$ before storing in air. The $\mathrm{Pd}$ content was $2.9 \mathrm{wt} \%$, as determined by atomic spectroscopy.

\subsection{ASAXS measurements}

Measurements were performed at the JUSIFA small-angle scattering beamline of the DORIS synchrotron radiation source at DESY (Hamburg) (Haubold et al., 1989). The contrast variation was performed at different energies (see Tables 1 and 2) near the Au $L(\mathrm{III})\left(E_{0}=11918 \mathrm{eV}\right)$ and the $\mathrm{Pd} K\left(E_{0}=24350 \mathrm{eV}\right)$ absorption edges. The energies are also indicated in Figs. 1 and 2, which show the energy-dependent part of the atomic scattering factor calculated using the method of Cromer \& Liberman (1970). Energies were calibrated using the measured $K$ absorption edges of Se $(12658 \mathrm{eV})$ and $\mathrm{Au}$ foils for the measurements of the Au-containing samples, and of the $K$ absorption edges of $\mathrm{Ru}(22117 \mathrm{eV})$ and $\mathrm{Pd}$ foils for the Pdcontaining samples.

Data were recorded using a two-dimensional gas-filled multiwire proportional counter, whose sensitivity map was determined by using an appropriate metallic foil (Se and Pd for measurements at the $\mathrm{Au}$ and Pd energies, respectively), and collecting for at least $12 \mathrm{~h}$ the isotropic fluorescence above its absorption edge and the small-angle scattering below it. By subtracting the latter from the former, a sensitivity map of the detector was obtained at the desired energy, which was used to correct the collected two-dimensional data. The dark current of the detector was measured at the beginning of the experimental session. Two-dimensional data were circularly averaged over rings at fixed $h$ values in order to give one-dimensional curves with the same abscissa for different energies. In order to have direct control of the statistical errors and of the possible variation with time of the source characteristics, a large number (up to 16) of short runs (1-3 min each) were collected, evaluated and then averaged. The background scattering was measured before each run by moving the sample out of the incident beam. Sample transmission was measured 


\section{Table 1}

Energy-dependent parts of the atomic scattering factor, $f^{\prime}$ and $f^{\prime \prime}$, near the Au $L$ (III)-edge energy, $E_{0}$, at the energies where ASAXS measurements have been carried out.

In the last column the average transmission is shown with uncertainties in parentheses.

\begin{tabular}{lllll}
\hline & $E(\mathrm{eV}) \pm 2 \mathrm{eV}$ & $f^{\prime}$ & $f^{\prime \prime}$ & Transmission \\
\hline$E_{1}$ & 11915 & -19.062 & 3.889 & $0.619(1)$ \\
$E_{2}$ & 11901 & -16.052 & 3.896 & $0.619(1)$ \\
$E_{3}$ & 11789 & -12.018 & 3.957 & $0.615(1)$ \\
$E_{4}$ & 11560 & -10.006 & 4.085 & $0.596(1)$ \\
\hline
\end{tabular}

with an X-ray sensitive diode before and after each run for $1 \mathrm{~min}$. Scattering from a reference scatterer (500 $\mu \mathrm{m}$ glassy carbon), used to calibrate the data in absolute units, was measured at the end of each run for $5 \mathrm{~min}$. Data were normalized to the primary beam intensity and corrected for the different absorption coefficients.

For the Pd sample, measurements at a sample-detector distance of $2.735 \mathrm{~m}$ were sufficient to give the whole required angular range, whereas, for the $\mathrm{Au}$ sample, measurements at two different distances ( $0.935 \mathrm{~m}$ and $3.635 \mathrm{~m})$ had to be matched.

In both samples studied, a single distribution of particles was not sufficient to describe the scattering intensity. For this reason a bimodal distribution was used,

$$
P(r)=\left[P_{1}(r)+K_{\mathrm{rel}} P_{2}(r)\right] /\left(1+K_{\mathrm{rel}}\right),
$$

where $K_{\text {rel }}$ is the ratio of the total number of smaller particles to that of the larger ones and $P_{i}(r)$ are normalized Schulz distributions,

$$
P(r)=\frac{1}{\Gamma(z+1)}\left(\frac{z+1}{\langle r\rangle}\right)^{(z+1)} r^{z} \exp \left(-\frac{z+1}{\langle r\rangle} r\right),
$$

where $z$ (with $z>-1$ ) is the form parameter of the distribution (Riello \& Benedetti, 1997).

Particles have been described by a spherical shape on the grounds of experimental considerations on the sample preparation procedure, which is likely to produce approximately spherical particles, and on TEM images (Polizzi et al., 2001). Yet, this particular choice does not affect the main features of the final results (Brumberger et al., 1996).

The catalyst powder was manually pressed into a cylindrical hole (6 mm diameter) of a metal sample holder and sealed with a nonscattering tape. A small lateral hole $(2 \mathrm{~mm})$, filled with a filter, connected the inner hole to the evacuated chamber. The sample thickness was $4 \mathrm{~mm}$ for both catalysts.

\section{Results and discussion}

A general experimental problem encountered with the investigated materials is related to the preparation of samples for the ASAXS measurements. Since metal-supported catalysts are usually powders, it is very important that the sample is mechanically stable during measurements. It was found that the pressure exerted under vacuum by the air trapped inside the powder was large enough to cause a visible swelling of the tape and rearrangement of the powder. This can change the density of the scattering material and the measured transmission, introducing errors in the data analysis. For this reason the original sample holder was modified by making a small lateral hole filled with a filter through which the trapped air can escape during chamber evacuation. Judging from the measured absorption values (see later), this sample holder seems to solve the problem of the sample stability.
Table 2

Energy-dependent parts of the atomic scattering factor, $f^{\prime}$ and $f^{\prime \prime}$, near the Pd $K$-edge energy, $E_{0}$, at the energies where ASAXS measurements have been carried out.

In the last column the average transmission is shown with uncertainties in parentheses.

\begin{tabular}{lllll}
\hline & $E(\mathrm{eV}) \pm 3 \mathrm{eV}$ & $f^{\prime}$ & $f^{\prime \prime}$ & Transmission \\
\hline$E_{1}$ & 24346 & -8.003 & 0.551 & $0.745(2)$ \\
$E_{2}$ & 24343 & -7.486 & 0.551 & $0.748(2)$ \\
$E_{3}$ & 24084 & -4.029 & 0.562 & $0.748(2)$ \\
$E_{4}$ & 23269 & -2.772 & 0.598 & $0.729(2)$ \\
$E_{5}$ & 23090 & -2.640 & 0.607 & $0.727(2)$ \\
\hline
\end{tabular}

The ASAXS signal is the difference between two measurements at two different energies, which are necessarily taken at a different time. When samples with small metal fractions are to be investigated, one is forced to increase the statistic quality of the data and to reduce as much as possible fluctuations in all experimental parameters that can occur during the collection time. To this aim, a measuring strategy consisting of the reiteration of short runs was considered to be much more appropriate than that of simply counting for longer times. Using a strategy of short-time runs, one is able to monitor all experimental variables (transmission, background, reference sample for the calibration of the scattering curves into absolute units) before and after each measurement, and to control the statistics of the experiment. It is clear that, for example, a short possible instability in the synchrotron incoming beam (position or intensity) would average in an integral measurement, whereas in a multiple-runs experiment such a fluctuation can be identified and the relevant run discarded. On the basis of the authors' experience, this is a very important check in a synchrotron radiation experiment, which uses highly complex instrumentation, and particularly in an ASAXS measurement, which is based on small differences owing to the energy dependence of the scattering intensity. Reiterate measurements of the transmission of the sample provide an idea of the stability, both of the sample itself and of the incoming beam. Since data are normalized to the energydependent transmission, this value must have an uncertainty at least smaller than the signal difference at the two energies studied, which is dependent on the difference in $f^{\prime}$ and on the metal content. In Figs. 3 and 4, transmission values (one before and one after each measurement) of different runs are shown for the four energies used near the Au absorption edge and for two different energies near the Pd edge (only two energies are shown for clarity in this case). In Tables 1 and 2 all average values are reported with their relevant uncertainties. The standard deviation of the transmission values is usually smaller than $1 \%$ of the measured values, so that averaging over ten runs $(20$ transmission values) yields an uncertainty lower than $0.2 \%$ (slightly higher for the Pd samples). The difference due to the change in energy is close to the statistical error for the energies in the neighbourhood of the edge, but it is clearly visible for most distant energies.

A further factor whose precision is crucial is the scaling factor for absolute intensity. This factor is calculated from the plateau present in the scattering intensity of a reference sample (glassy carbon), which is previously calibrated using a rotating anode. Thus, the scaling factor is a measure of the primary beam intensity and, in fact, a direct correlation was found with the incoming intensity measured using a $\mathrm{NaJ}$ detector. However, strongly diverging values were often found during the first half hour after injection of a new synchrotron beam, probably owing to a settlement of the beam position. The intensity of a synchrotron beam is continuously decreasing with time, so that measurements of the glassy carbon must be as close as 
possible in time to that of the sample, but measurements too close to a new injection should be discarded. The measuring strategy proposed in this work allows the former condition to be satisfied and a few measurements to be discarded with a minimum waste of beam time.

Short-time oscillations of the incoming beam could also give rise to errors in the value of the measured transmission, since this is determined by two successive intensity measurements (with and without sample). However, with the adopted measuring strategy, the influence of such instabilities is reduced, and, in fact, no correlation was found between transmission values and the factor for absolute intensity, which should exclude this kind of error.

Let us finally look at the statistical errors on the curves obtained as an average of the different experimental runs by comparing curves at different energies. In order to have a direct indication of the real experimental errors, each run was evaluated separately. Averages and

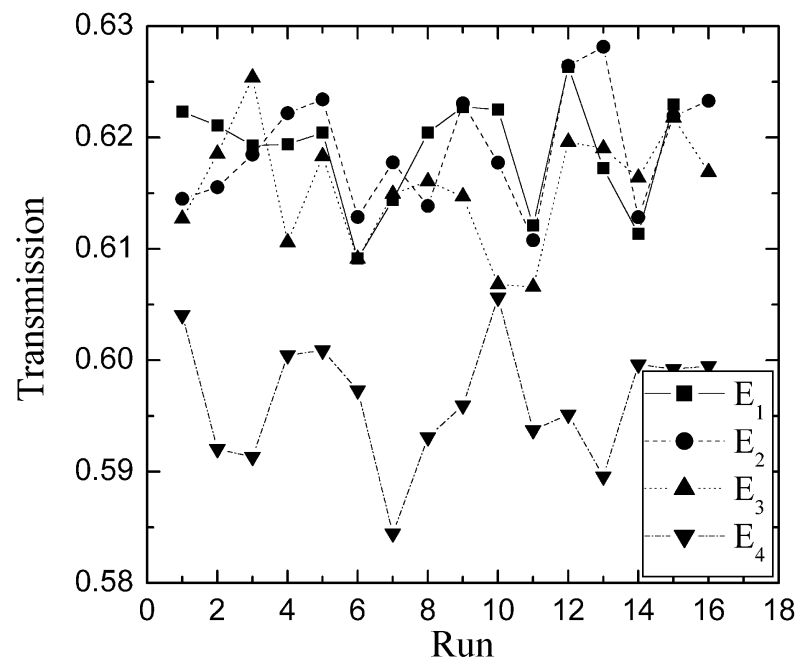

Figure 3

Transmission values of the $\mathrm{Au}(0.2 \mathrm{wt} \%) / \mathrm{C}$ sample measured before and after each of eight different experimental runs at different energies near the $\mathrm{Au}$ absorption edge.

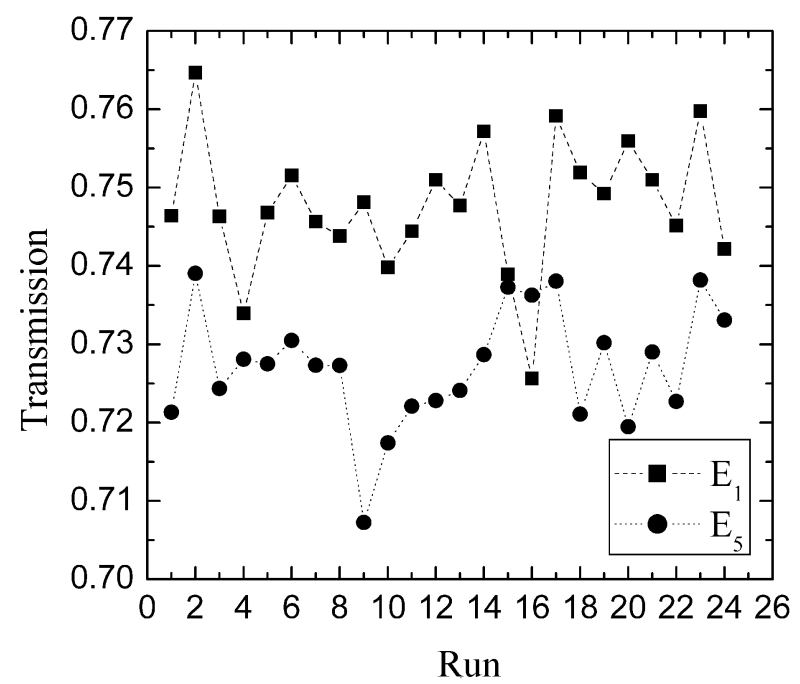

Figure 4

Transmission values of the $\mathrm{Pd}(2.9 \mathrm{wt} \%) / \mathrm{SiO}_{2}$ sample measured in the different experimental runs at different energies near the Pd absorption edge. Only two energies are shown for clarity. standard deviations were then worked out for each single data point of all scattering curves measured at the same energy. In Fig. 5 the average scattering intensities of eight runs for the two most distant energies $\left(E_{4}\right.$ and $\left.E_{1}\right)$ measured at the longer sample-detector distance is shown for the Au-containing sample, together with the relevant standard deviations of the average. The difference between the two curves is very small, but the insets show that they are clearly separated by more than one standard deviation, at least until $h$ values of about $1.6 \mathrm{~nm}^{-1}$, and tend to overlap only in the very end of the angular range, where the intensity is very small. The curve obtained by subtracting the $E_{1}$ curve from the $E_{4}$ curve (the subtracted curve) with error bars calculated from the relevant standard deviations and the best-fit with equation (1) has already been published in an earlier paper (Benedetti et al., 1997, 1999). It is important to note that the scattering difference obtained by other energy pairs gradually decreased with decreasing energy difference, showing the reliability of the data. Two different populations of particles were found from the quantitative analysis of these curves: one with average diameters of $14 \mathrm{~nm}$ and the other consisting of small particles with an average size of $2 \mathrm{~nm}$. Furthermore, a very interesting finding was that the small particles are an overwhelming majority, being three times the weight of the larger ones. Even if these values are subjected to large errors, because the scattering owing to small particles takes place at larger $h$ values where the data are less accurate, the presence of a large population of particles smaller than $3 \mathrm{~nm}$ has been confirmed by a later study using Rietveld analysis (Riello et al., 1998.) The total weight fraction has now been calculated and is compared with the value obtained by atomic absorption in Table 3. As explained in the

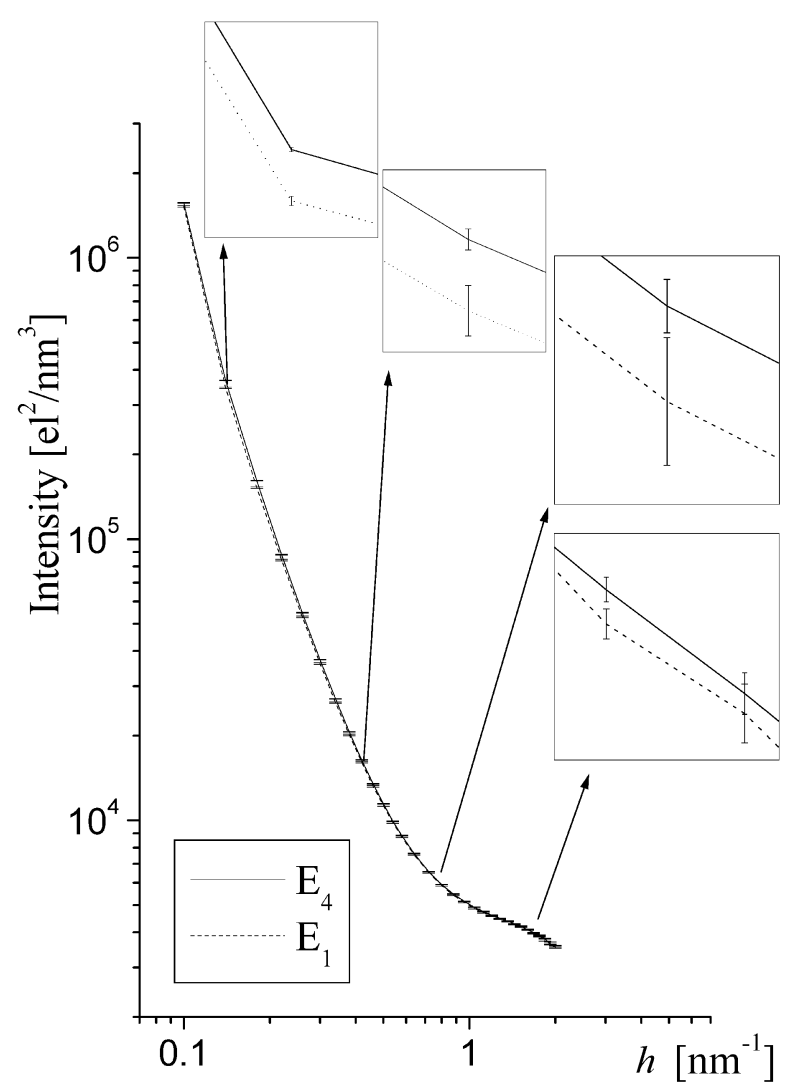

Figure 5

Scattering curves of the $\mathrm{Au}(0.2 \mathrm{wt} \%) / \mathrm{C}$ sample at two different energies $\left(E_{1}\right.$ and $E_{4}$, sample-detector distance $=3.635 \mathrm{~m}$ ). Insets show different enlarged regions. 
theoretical section, the range of values indicated for the ASAXS result indicates the two limit assumptions for the electron density of the matrix. It can be seen that the uncertainty due to the latter choice is small and that the ASAXS value agrees with the result obtained by atomic absorption.

Discussing now the measurements of the Pd-containing samples, it must be noticed that, from the experimental point of view, measurements at the $\mathrm{Pd}$ edge present some further problems in addition to those encountered at the Au edge.

First, the difference in the scattering factor at two different energies close to the $\mathrm{Pd} K$ edge is quite small: the highest difference at the measured energies is just 5.4 electrons, in contrast to the 9 electrons at the $\mathrm{Au} L(\mathrm{III})$ edge. This means that the signal level is much lower and that the sensitivity to Pd of the technique is almost half of that for $\mathrm{Au}$.

Also, the energy resolution of the monochromator at JUSIFA is $\Delta E / E<2 \times 10^{-4}$, so that at the Pd edge an uncertainty of the order of a few eV can be expected.

In Fig. 6 the two scattering curves for the Pd-containing sample for $E_{1}$ and $E_{5}$ obtained by averaging over 12 runs are shown together with the relevant standard deviations. It can be seen that data are separated by more than one standard deviation, at least until $h$ values of about $2 \mathrm{~nm}^{-1}$, and that at higher $h$ values a crossover of the curves occurs. Such a crossover has already been described in similar measurements (Schliermann et al., 2000) and can be due to different effects such as a very small residual fluorescence background close to the absorption edge, to second-order (mixed terms) resonant scattering or to a special electronic configuration (e.g. oxide film).

The final subtracted curve is shown in a double logarithmic scale in Fig. 7, together with error bars and the best-fit obtained using

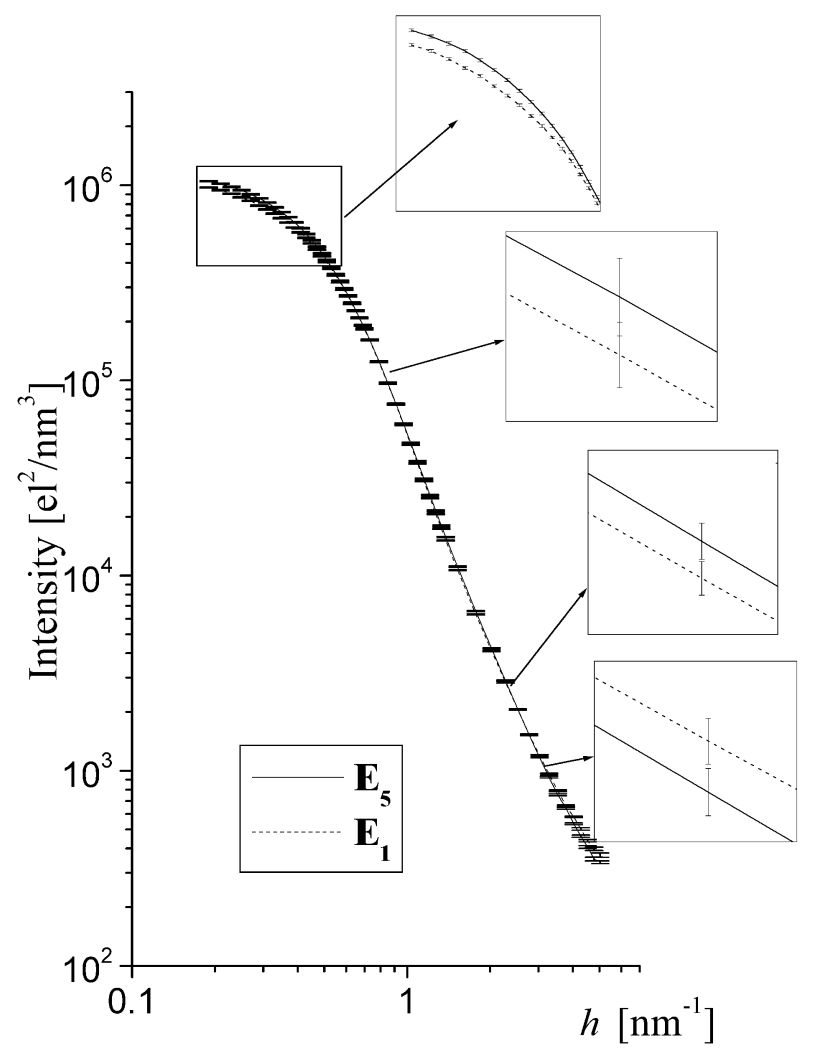

Figure 6

Scattering curves of the $\mathrm{Pd}(2.9 \mathrm{wt} \%) / \mathrm{SiO}_{2}$ sample at two different energies. Insets show different enlarged regions.
Table 3

Total metal fraction obtained by atomic absorption and by SAXS.

The range indicated in the ASAXS results refers to two different choices for the electron density of the matrix (see text).

\begin{tabular}{lll}
\hline Sample & Atomic absorption & ASAXS \\
\hline $\mathrm{Au} / \mathrm{C}$ & $0.20(1) \mathrm{wt} \%$ & $0.18 \%-0.21 \%$ \\
$\mathrm{Pd} / \mathrm{SiO}_{2}$ & $2.9(2) \mathrm{wt} \%$ & $2.3 \%-3.1 \%$ \\
\hline
\end{tabular}

equation (1). Notwithstanding the large uncertainties and the aforementioned experimental problems, the final results that are drawn from this curve are quite reasonable and in very good agreement with the findings of other techniques (TEM, WAXS, EXAFS, absoprtion spectroscopy), as shown elsewhere (Polizzi et al., 2001). The main results of this analysis were: (i) the presence of two populations of particles with average values of $3 \mathrm{~nm}$ and $13 \mathrm{~nm}$; (ii) smaller particles represent some $80 \%$ of the whole metal; (iii) quantitative analysis of the Pd content gave 2.7 (4) wt \%, in accordance with the value obtained by atomic spectroscopy (see Table 3). Again, the range of the ASAXS value indicates the two limit assumptions for the electron density of the matrix. The uncertainty in the quantitative determination for the Pd sample is larger than for the Au sample because the difference between the electronic density of the metal and that of the support is smaller for Pd and silica than for $\mathrm{Au}$ and carbon. One could think that the best value to be used for evaluating the electronic density contrast should be the average electronic density of the sample. Yet, with this choice a metal content of $2.4 \%$ was found, whereas the atomic absorption value was much

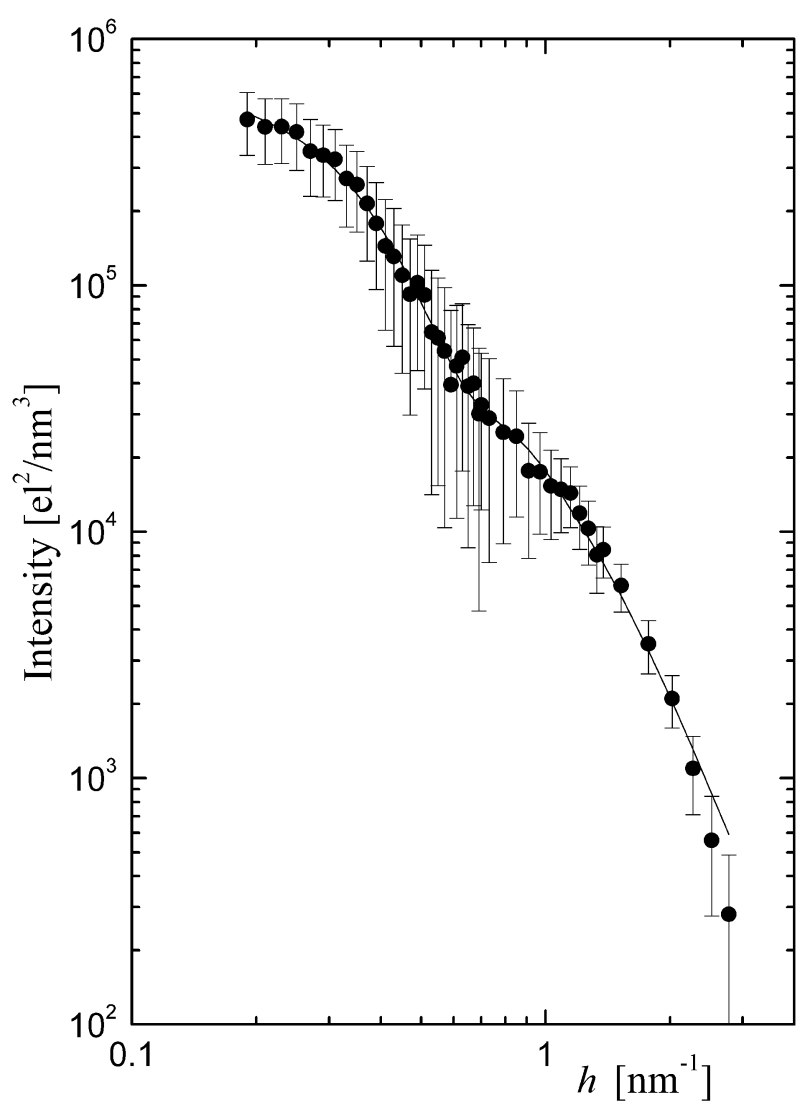

Figure 7

Final scattering curve of the $\mathrm{Pd}(2.9 \mathrm{wt} \%) / \mathrm{SiO}_{2}$ sample in a double logarithmic plot. The continuous line is the best-fit obtained using equation (1). 
closer to the value obtained using the electronic density of bulk silica. Apparently, metal particles are, on average, surrounded more by silica than by pores.

\section{Conclusions}

It was shown that a measuring strategy consisting of reiterated shorttime runs with frequent control of the experimental parameters is recommendable in the case of metal-supported catalysts with low metal load in order to reach a sufficient control of the statistics of the data. By this method, gold content as low as $0.2 \mathrm{wt} \%$ and palladium content of $3 \mathrm{wt} \%$ could be investigated with success. The detection limits for palladium are higher, mainly due to its smaller electronic contrast.

This work was supported by the TMR Contract ERBFMGECT950059 of the European Community. Financial support from $60 \%$ MURST is also acknowledged. The authors are grateful to one of the referees for his/her sharp remark concerning equation (4), that inspired the footnote.

\section{References}

Benedetti, A., Bertoldo, L., Canton, P., Pinna, F., Goerigk, G., Riello, P. \& Polizzi, S. (1999). Catal. Today, 49, 485-489.

Benedetti, A., Polizzi, S., Riello, P., Pinna, F. \& Goerigk, G. (1997). J. Catal. 171, 345-348.

Brumberger, H., Goodisman, J., Ramaya, R. \& Ciccariello, S. (1996). J. Appl. Cryst. 29, 526-530.

Cromer, D. T. \& Liberman, D. (1970). J. Chem. Phys. 53, 1891-1898.

Guinier, A. \& Fournet, G. (1955). Small-Angle Scattering of X-rays, p. 19. New York: Wiley.

Haubold, H.-G., Gruenhagen, K., Wagener, M., Jungbluth, H., Heer, H., Pfeil, A., Rongen, H., Brandenburg, G., Moeller, R., Matzerath, J., Hiller, P. \& Halling, H. (1989). Rev. Sci. Instrum. 60, 1943-1946.

Haubold, H.-G. \& Wang, X. H. (1995). Nucl. Instrum. Methods Phys. Res. B, 97, 50-54.

Haubold, H.-G., Wang, X. H., Goerigk, G. \& Schilling, W. (1997). J. Appl. Cryst. 30, 653-658.

Haubold, H.-G., Wang, X. H., Jungbluth, H., Goerigk, G. \& Schilling, W. (1996). J. Mol. Struct. 383, 283-289.

Polizzi, S., Riello, P., Balerna, A. \& Benedetti, A. (2001). Phys. Chem. Chem. Phys. 3, 4614-4619.

Rasmussen, F. Berg, Molenbroek, A. M., Clausen, B. S. \& Feidenhans, R. (2000). J. Catal. 190, 205-208.

Riello, P. \& Benedetti, A. (1997). J. Chem. Phys. 106, 8660-8663.

Riello, P., Canton, P. \& Benedetti, A. (1998). Langmuir, 14, 6617-6619.

Schliermann, T., Glora, M., Pröbstle, H., Petričević, R. \& Fricke, J. (2000). HASYLAB Annual Report, pp. 449-450. HASYLAB, DESY, Hamburg, Germany. 